\title{
Reliable Redundancy with Memristive-Biosensors to achieve Statistical Significance in Immunosensing
}

\author{
A. Lotfi and D. Demarchi \\ Department of Electronics \\ and Telecommunications, PoliTO \\ 10129 Torino, Italy \\ Email: ardalan.lotfi@epfl.ch
}

\author{
F.Puppo, G. De Micheli and S. Carrara \\ Integrated System Laboratory, \\ EPFL \\ 1015 Lausanne, Switzerland \\ Email: francesca.puppo@epfl.ch
}

\author{
M.-A. Doucey \\ Ludwig Center for Cancer Research, \\ UNIL \\ 1066 Epalinges, Switzerland \\ Email: marie-agnes.doucey@unil.ch
}

\begin{abstract}
In this paper, we have investigated the redundancy in array of Memristive-Biosensors and find optimum number for devices to accomplish reliable biodetection. Our results lead less expensive sensor and reduce the low-reproducibility of this memristive method for the detection of rabbit antigen. Several experiments have been performed with 17 memristive biosensors in several conditions. These conditions give us relevant informations about the overall behavior of memristor biosensor's array, after functionalization with antibody and exposure to antigen. The statistics made on different conditions, related to the standard deviation and the mean value of voltage gap, prove that such a sensor, with a minimum memristive biosensors in array, can be counted as reliable sensor for the detection of antigen.
\end{abstract}

\section{INTRODUCTION}

In recent years, electronic detection of biomolecules has become one of the most hot research topics in nanotechnology. New developments have paved the way to a large number of novel nano-scale devices of highly promising properties for electrochemical sensor and biosensor applications[1], [2], [3], [4], [5]. In particular, nanowires have become the focus of intensive research because of their unique properties and their potential for fabrication of nanosensors which can provide fast, low-cost, and high-throughput analysis of biological processes. Indeed, the electrical properties of nanowires are strongly influenced by minor perturbations [6], [7] because of their high surface-to-volume ratio. Thus, these properties offer new capabilities not available in larger scale devices. Unlike 2-D thin films, the charge accumulation or depletion in the 1-D nanostructure takes place in the bulk of the structure thus giving rise to large changes in the electrical properties that potentially enable the detection of a single molecule [8]. Up to now, this property of the 1-D nanostructures has been shown to provide a sensing modality for label-free and direct electrical readout when the nanostructure is used as a semiconducting channel of a chemiresistor or field-effect transistor [9], such as in gas sensors [10] and Ion-Sensitive Field-Effect Transistors (IS-FETs) for cancer markers or DNA [11] detection.

Recently, a completely new insight for biosensing based on the memristive effect of functionalized Schottky-barrier silicon nanowires in dry environment has been reported [12]. Because of the nano-scale of the fabricated geometries [13], silicon nanowire devices have been discovered to show hysteretic properties reminiscent of the memristive devices [14]. In these devices, the memory effect depends on charge carriers rearrangement at the nanoscale as due to external perturbation.
Moreover, nanofabricated memristive silicon nanowires, functionalized with biomolecular films, have been demonstrated to sense varying concentrations of protein solutions thanks to corresponding variations of the hysteresis curve parameters[12]. The air humidity has revealed one important factor strongly affecting the nanowire biosensor performances in dry environment and Carrara et al. have already demonstrated the role of the water shell adsorbed from the surrounding humid environment to the surface of a layer of rather small organic molecules, supposed not to have internal water [15]. However, the low-reproducibility of any biosensing method based on silicon nanowires is a challenging issue due to the fact that extremely small nano-sensors are highly sensitive to any small environmental variation. On the other hand, the low reproducibility due to the variation in properties of individual devices is a very well known problem met several times in other disciplines of the technology. It's worth to mention that the concept of redundancy has been especially developed in electronics to keep such variations under control. In fact, the concept of redundancy has been already successfully proposed for several other domains such as programmable logic devices[23], dynamic random access memory[24], to minimize the coding digits per message[25], failures selection[26], and other domains.

In particular, the aim of this study is to identify an optimum number of the memristive nanowires in order to accomplish simultaneously collecting reliabe biodata, less expensive sensor and overcoming the low-reproducibility of this memristive method for the detection of rabbit antigen. To achieve such a goal, several experiments have been performed with 17 memristive nanowires, and a couple of conditions were applied to the obtained data. These conditions give us relevant information about the overall behavior of memristor nanowire, after functionalization with antibody and exposition to the cognate antigen.

\section{EXPERIMENTAL APPARATUS}

\section{A. Memristive Nanowire Fabrication}

Memristive silicon nanowires are fabricated through a topdown approach performed on a Silicon-On-Insulator (SOI) wafer with a $350 \mathrm{~nm}$ thick device layer. Puppo et al. have already explained the critical steps of process flow[16]. The dimension of utilized memristive silicon nanowires in the performed experiments are $470 \mathrm{~nm}$ in length and $30 \mathrm{~nm}$ in width. 


\section{B. Functionalization of the Memristive-biosensors}

To functionalize the Memristive-Biosensors with antibody, the self-assembly methode has been used. The devices were functionalized by covalent attachement of anti-rabbit monoclonal antibody through a cross linker named GPTS[17]. This was performed using a modification of the process described in cited article[18]. Figure 1(a) shows functionalized MemristiveBiosensors with antibody. The crosslinker, GPTS, has linked the antibody to the surface of the nanowires having natural oxide layer.

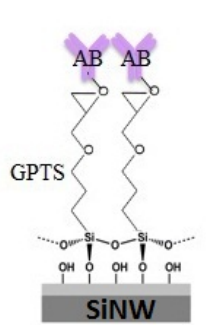

(a)

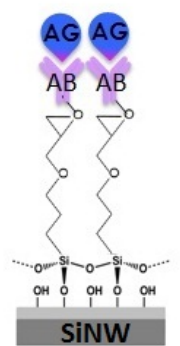

(b)
Fig. 1. (a) Memristive-Biosensors functionalized with antibody, and (b) after exposure to the rabbit antigen.

\section{Sensing protocol}

The functionalized Memristive-Biosensors were exposed to a concentration of the target molecules by drop casting a $300 \mu 1$ drop of $10 f M$ antigen solution in PBS on top of the device et al.[16]. The sensor was kept incubated in exogenous rabbit antigen solution for 1 hour at room temperature. After the incubation, the sensor was washed in PBS to remove the unreacted antigen molecules, gently dried under nitrogen flow and then measured in dry conditions[19]. $\mathrm{I}_{d s}-\mathrm{V}_{d s}$ characteristics were acquired in a Signatone H-100 Probe Station and by using a Keithley 6430 Sub-Femtoamp SourceMeter. The back-gate was kept grounded. Changes of the hysteretic voltage gap of the sensor were monitored prior to and after antibody functionalization, and then after exposure of the sensor to the antigen. All the acquisitions were performed under controlled conditions: at the relative humidity $(\mathrm{rH})$ of $50 \%$ and a temperature of $21^{\circ} \mathrm{C}$. The proposed improved sensitivity in dry condition [20], [21] was ensured by an increased Debye length deriving from the absence of counterions from the bulk electrolytic solution [22].

\section{EXPERIMENTAL DATA AND RESULTS}

The voltage gap shown by the Memristive-Biosensors has been measured at all stages of the experimental procedures as experiments explained in Section II-D. In several cases the fabricated sensors do not follow the expected behavior and voltage gap is not changed regularly (figure 2). The expected behavior is that voltage gap increases after antibody functionalization and shrinks after antigen uptake. This variation has been caused by the extremely high sensitivity of the Memristive-Biosensors and results in a high variation and that can be overcome by increasing the number of measurement.

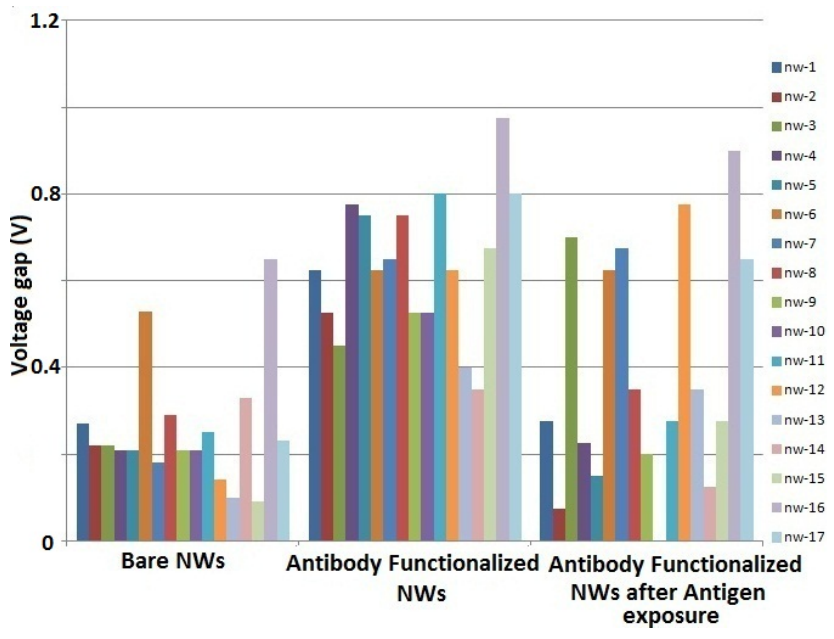

Fig. 2. Measured value of voltage gap for each Memristive-Biosensor at 3 different stages - bare Memristive-Biosensor, after functionalization with antibody and after exposing the sensor to the target molecule.

\section{A. Statistical analysis of the measurements}

A two tailed paired T-test was applied to groups of biodata. For the sake of simplicity, each biodata set was named as a group: Group A (Group of bare Memristive-Biosensors), B (Group of antibody functionalized Memristive-Biosensors) \& $\mathrm{C}$ (Group of antigen-exposed Memristive-Biosensors).

1) Two tailed paired T-test for group $A \& B$ : The result of T-test for the group A and B is significant, and it proves that change in voltage gap doesn't happen randomly. We can simply extract from this T-test result that the impact of functionalizing Memristive-Biosensors with antibody is the increase in the voltage gap. The T-test for these set of data rejects the null hypothesis and the null hypothesis means that the Memristive-Biosensors behave randomly. The null hypothesis has been rejected by T-test. Furthermore, because of repeating many experiments like what presented in this work, it can be claimed that the increase in the voltage gap is resulted by functionalization of the Memristive-Biosensors with mentioned antibody. The result of T-test on group A and $\mathrm{B}$ has been presented in table I.

\section{TABLE I. T-TEST RESULT APPLIED TO GROUP A\&B}

$$
\begin{array}{|c|c|}
\text { T-test result } & \text { Rejection of null hypothesis } \\
\text { Probability } & 2.1911 \mathrm{e}-07 \\
\text { Confidence } & \text { (the less ,the stronger probability of rejection null hypothesis } \\
\text { interval } & {[0.2814,0.4580]} \\
\text { tstat } & -8.9224
\end{array}
$$

2) Two tailed paired T-test for group $B \& C$ : The result of T-test for the group B and $C$ is significant, and it shows that the overall behavior of the Memristive-Biosensors is convergent again. It proves that changes in voltage gap do not happen randomly. Simply speaking, it can be concluded from the Ttest result that the majority of the devices show voltage gap decreasing after uptaking the antigen. The T-test for these set of data rejects the null hypothesis. The null hypothesis is that the Memristive-Biosensors behave randomly. The null hypothesis has been rejected by T-test. Furthermore, because of repeating 
many experiments like what presented, it can be claimed that the decrease in the voltage gap is resulted by uptaking the antigen through antibody. The result of T-test on group A and $\mathrm{B}$ has been presented in table II.

TABLE II. T-TEST RESUlT APPLIED TO GROUP B\&C

$$
\begin{array}{c|c|}
\text { T-test result } & \text { Rejection of null hypothesis } \\
\text { Probability } & 0.0043 \\
\text { Confidence } & \text { (the less ,the stronger probability of rejection null hypothesis } \\
\text { interval } & {[-0.3834,-0.0866]} \\
\text { T-test value } & -8.9224
\end{array}
$$

By considering the result of the T-test between each couple of Memristive-Biosensor groups, the changes in mean value of the voltage gap can be considered as a signal for successful antibody functionalization and detection of rabbit antigen. The average voltage gap shown by set of Memristive-Biosensors increased after functionalization with antibody and decreased after exposing the rabbit antigen to the sensor.

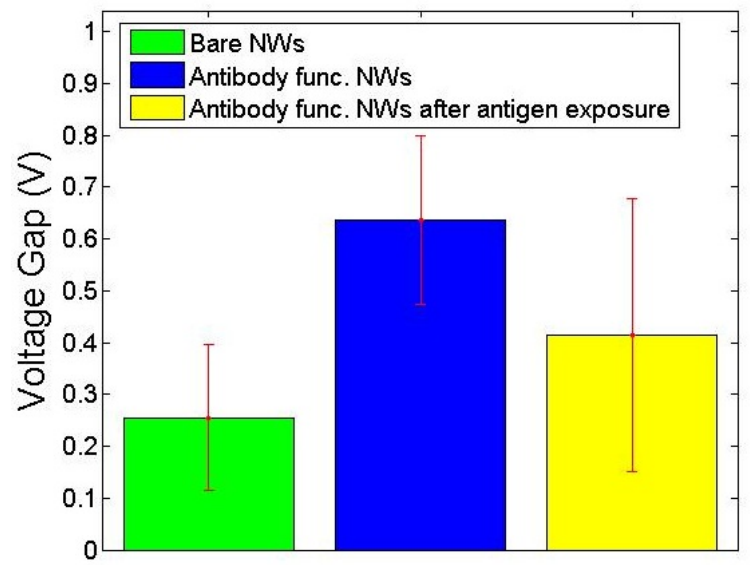

Fig. 3. Each column shows the average voltage gap shown by MemristiveBiosensors in different stage of experiment-bare Memristive-Biosensors, after functionalization with antibody and after exposing the sensor to the target molecule.

\section{DATA ANALYSIS AND RESULTS}

\section{A. Applied conditions}

16 Memristive-Biosensors have been used to detect rabbit antigen in PBS. It is desirable to randomly choose a number of Memristive-Biosensors and check their average voltage gap and repeat this process to obtain standard deviation of mean voltage gap value. To explain the applied method, the maximum number of combinations which can be chosen from space of 16 Memristive-Biosensors have been present in figure 4.

It is possible to randomly choose a set including \#NUM of Memristive-Biosensors and repeat the 3 steps process for known cycle presented in figure 4(red columns). The method has three key steps. To start integrating statistics with our data, the steps presented below should be followed till satisfying the loop condition between step1 and step3. The steps are depicted in figure 5 .

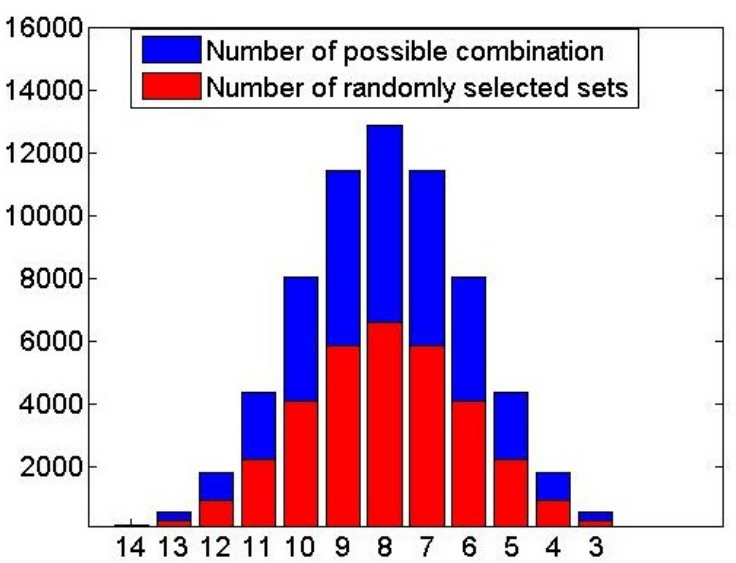

Fig. 4. The amount of possible combination that randomly can be selected is presented by blue, the number of randomly selected set which we select is presented in red color.

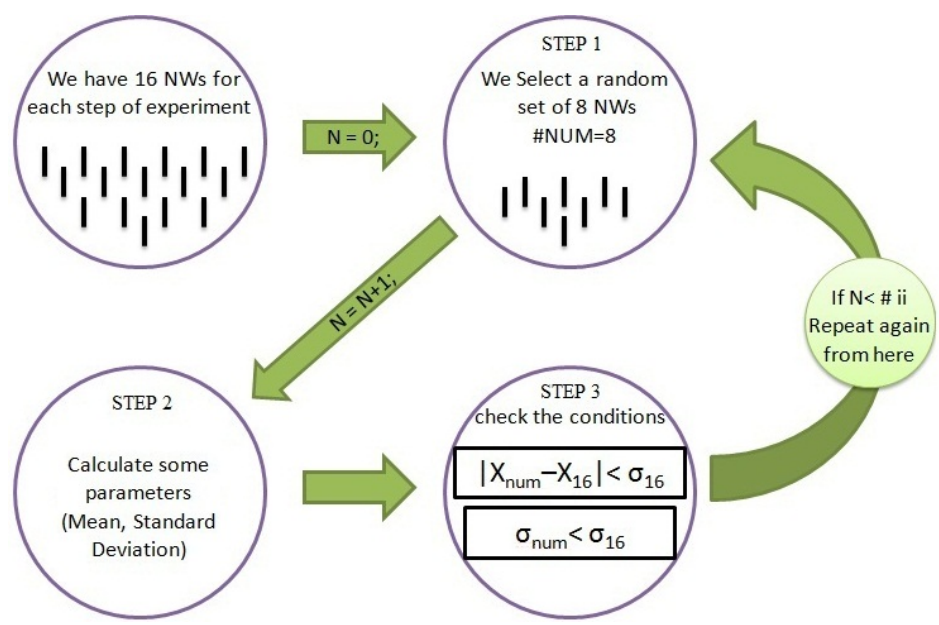

Fig. 5. The graph shows percentage of the sets with \#Num of MemristiveBiosensors which passed the T-test successfully. The pair sets are MemristiveBiosensors functionalized with antibody and bare Memristive-Biosensors

STEP 1 : \#NUM of Memristive-Biosensors, a certain number obtained from figure 4 , is randomly selected among all the 16 Memristive-Biosensors. The program counts them as a random set which contains \#NUM Memristive-Biosensors.

STEP 2 : the mean value and standard deviation of the voltage gap are calculated and the program stores theirs value.

STEP 3 : two conditions are applied to the computed value. If the number of cycles doesn't reach the saturation, the loop starts again from step 1 . The loop between step $1,2 \& 3$ can not be repeated more than the numbers presented in red column of figure 4 .

The program aim is to count the number of randomly selected sets that satisfies the conditions applied in step 2 and the results are shown as a percentage for the group containing \#NUM Memristive-Biosensors. In the second Step of the loop, two conditions are applied. They are explained in following: 
Condition $1 \quad\left\|X_{N U M}-X_{16}\right\|<\sigma_{16}$

$X_{N U M}$ is the mean voltage gap of the set including \#NUM Memristive-Biosensors. $X_{16}$ is the mean voltage gap of the set including 16 Memristive-Biosensors. $\sigma_{16}$ is the standard deviaCondition 2 ton of the set including 16 Memristive-Biosensors. $\sigma_{N U M}$ is the standard deviaton of the all sets including \#NUM Memristive-Biosensors.

\section{B. Statitics on the number of Memristive-Biosensors and their T-test results}

To start extracting the results of the T-test, the number of Memristive-Biosensors included in set has been increased. What is interesting to present is how the percentages change in Figure 6 and are affected while the number of MemristiveBiosensors in the set decreases. By looking at Figure 6 (Ttest on group $A \& B$ ), it can be seen that all the sets with 6 Memristive-Biosensors or greater completely has passed the T-test. Thus, with 6 or any greater number of MemristiveBiosensors the successful attachment of antibody to the Memristive-Biosensors can be distinguished from the change in voltage gap. As a result, the issue of low-reproducibility of the device has been overcome.

The principles are the same to the previous pair groups. As it can be seen from figure 7, the amount of the sets passing the T-test successfully is slowly diminishing while the number of Memristive-Biosensors in the set decreases. In figure 7, there are two interesting categories. The first category are the sets with more than 7 Memristive-Biosensors. The second category are sets with more 12 Memristive-Biosensors. Based on figure 7 , it can be claimed that the sets with more than 12 MemristiveBiosensors are completely passing T-test applied on group B and $\mathrm{C}$. This means that if the set of Memristive-Biosensors contains more than 12 Memristive-Biosensors the results are reliable and convergent. As a result, the uptaking of antigen is resulted in voltage gap decreasing. For the group with more than $7 \mathrm{NWs}$, the result of T-test shows that the probability of passing T-test with 8 nanorwires is more than $50 \%$. This means if the experiment is handled with $8 \mathrm{NWs}$, the voltage gap change of NWs coincide to each other with the probability greater than $\frac{1}{2}$. However, the results would never be deceiving.

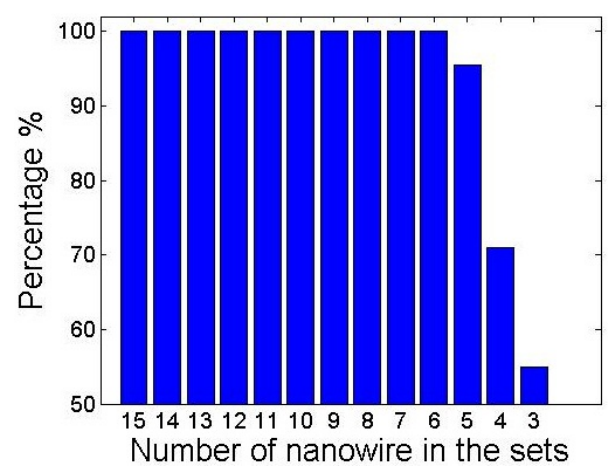

Fig. 6. Percentage of the sets with \#Num of Memristive-Biosensor which passed the T-test successfully. The pair sets are Memristive-Biosensors functionalized with antibody and bare Memristive-Biosensors

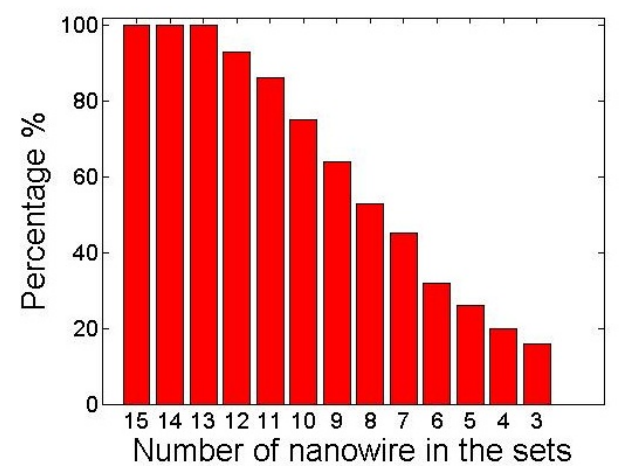

Fig. 7. Percentage of the sets with \#Num Memristive-Biosensors which passed the T-test successfully.The pair sets are Memristive-Biosensors functionalized with antibody and Memristive-Biosensor after exposing to the rabbit Antigen

\section{Statictics on the mean of bare Memristive-Biosensors}

The statistic results on bare Memristive-Biosensors, figure 8 , show that the minimum number of bare MemristiveBiosensors which behave mostly similar to the 16 MemristiveBiosensors is 6 . Therefore, it can be derived from the statistics that acquired data from 6 or any greater number of MemristiveBiosensors is reliable for the bare Memristive-Biosensor stage. For antibody functionalized NW's , the results of statistics, figure 8, show that the minimum number of MemristiveBiosensors which behave near similar to the 16 MemristiveBiosensors is 6 . Thus, it can be concluded from the statistics that acquired data from 6 or any greater number of MemristiveBiosensors is reliable for the antibody functionalized stage.

The statistic results on the mean voltage gap value of antigen-exposed Memristive-Biosensors have been presented in figure 10. This shows that the minimum number of Memristive-Biosensors which behave near similar to the 16 Memristive-Biosensors is 6. As a result, it can be concluded from the statistics that acquired data from 6 or any greater number of Memristive-Biosensors is reliable for the antigen exposing stage.

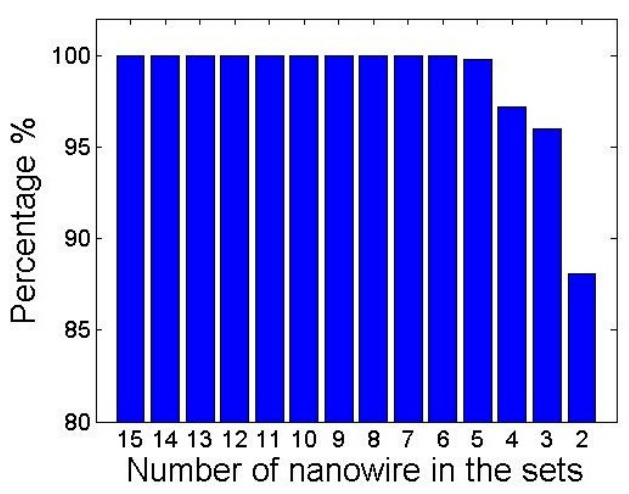

Fig. 8. Percentage of bare sensor sets containing \#NUM MemristiveBiosensors which satisfies the applied condition 1 on their means. 


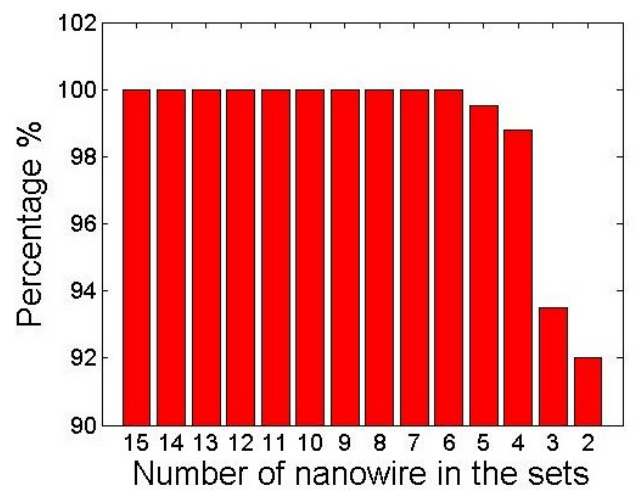

Fig. 9. Percentage of antibody funcionalized Memristive-Biosensors set containing \#NUM Memristive-Biosensors which satisfies the applied condition on their mean values, condition 1 .

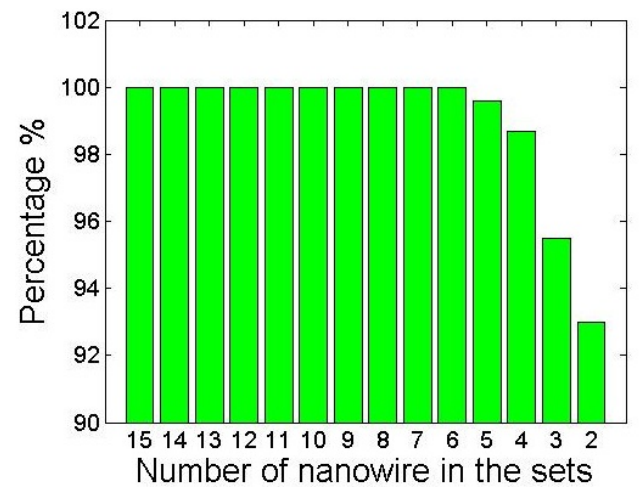

Fig. 10. Percentage of nanowire sets exposed to antigen containing \#NUM Memristive-Biosensors satisfying the applied condition 1 on their means.

\section{Statictics on the standard deviation of bare Memristive- Biosensors}

Figure 11 explicitly shows that if the number of bare Memristive-Biosensors increases from 6 to 13, there is not any significant change in the percentage of the sets satisfying the condition on standard deviation. As a result, it can be concluded that 6 is an optimum for the number of MemristiveBiosensors in the bare stage. The story would be the same for the antibody-functionalized Memristive-Biosensors. Figure 12, it can be seen that if the number of antibody-functionalized Memristive-Biosensors increases from 6 to 13, there wouldn't be a significant change in the percentage of the sets satisfying the condition on standard deviation. Thus, it can be concluded that the 6 is an optimum for the number of MemristiveBiosensors in the second stage of the experiment, measuring the voltage gap of antibody-functionalized MemristiveBiosensors. It is explicit in figure 13 that we have the same conclusion for the antigen-exposed Memristive-Biosensors. It can be derived that if the number of Memristive-Biosensors increases from 6 to 13, there is no significant change in the percentage of the sets satisfying the condition. It is inferred that the 6 could be an optimum for the number of MemristiveBiosensors in detection of target bio-molecule, rabbit antigen.

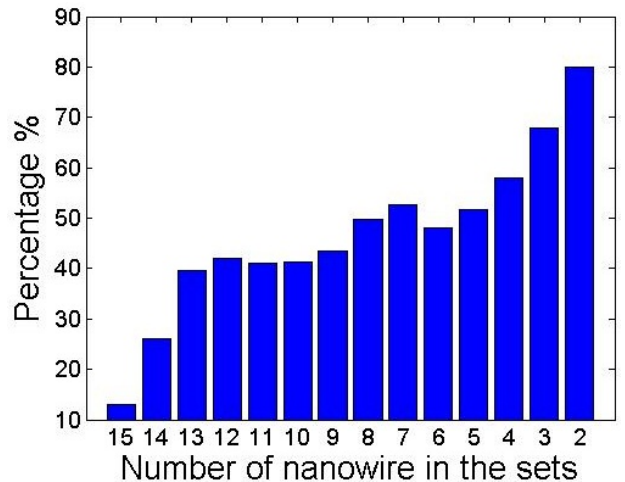

Fig. 11. Percentage of bare Memristive-Biosensors set containing \#NUM Memristive-Biosensors which satisfies the applied condition on their standard deviation, condition 2

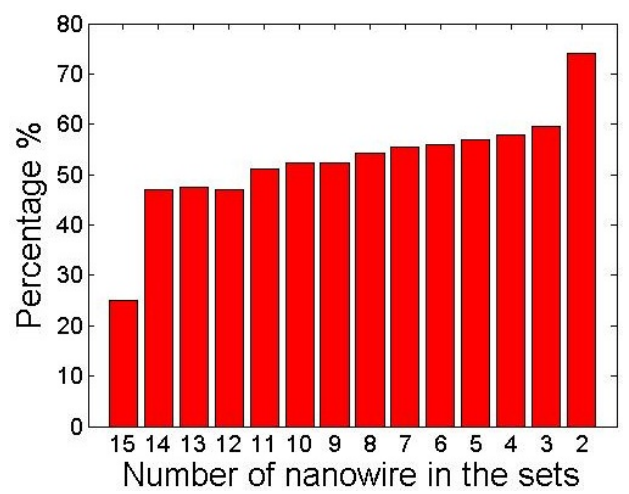

Fig. 12. Percentage of functionalized sensor sets containing \#NUM Memristive-Biosensors which satisfies the applied condition 2 on their standard deviation.

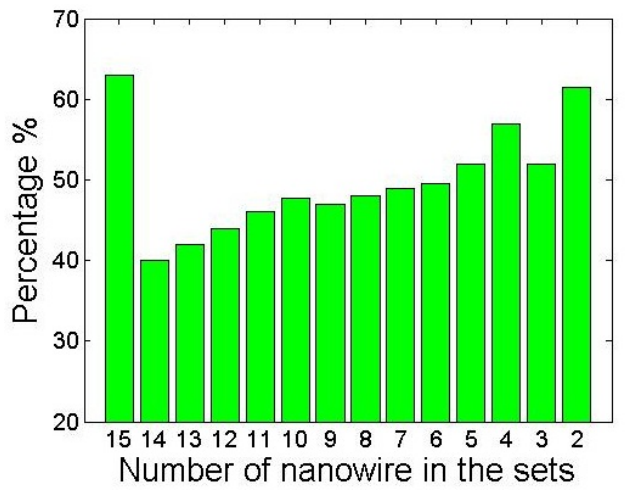

Fig. 13. Percentage of sensor sets exposed to antigen that contain \#NUM Memristive-Biosensors satisfying the applied condition 2 on their standard deviation. 


\section{CONCLUSION}

By analyzing the data of 17 Memristive-Biosensors and applying mentioned conditions on them as discussed in this work, an optimum number of Memristive-Biosensors can be reported in order to overcome the low reproducibility of the detecting antigen with memristive silicon nanowire, MemristiveBiosensor. To reduce the low reproducibility of the device for bare Memristive-Biosensor voltage gap, the experiment should consist of at least 6 Memristive-Biosensors. This mimnimum number would be the same for the rest of experiment stages which can satisfy the whole conditions applied during extracting statistic fact about data. For proposing the optimum number, one more limitation should be taken into the account and it is about the T-test results on experiment stage pairs. To have a truthful interpretation of the data extracted by comparing the average voltage gap obtained from bare MemristiveBiosensor and antibody functionalized Memristive-Biosensor, the number of Memristive-Biosensors in experiment should be equal or greater than 6 . The comparison between the antibody functionalized Memristive-Biosensors set and the antigen-exposed Memristive-Biosensors is similar to what has been presented for the previous expression. The minimum number of Memristive-Biosensors for having significant T-test results is 8 and significant T-test result gives us authority to compare average voltage gap of different experiment stages and to conclude if the detection of antigen was successful or not.

\section{ACKNOWLEDGMENT}

This research has been supported by the Swiss Multidisciplinary SNF project CR32I3/156915.

\section{REFERENCES}

[1] J. Hubalek, et al., Spectrometric and voltammetric analysis of urease nickel nanoelectrode as an electrochemical sensor, Sensors, vol. 7, pp. $12381255,2007$.

[2] U. Yogeswaran, et al., Pinecone shape hydroxypropyl-cyclodextrin on a film of multi-walled carbon nanotubes coated with gold particles for the simultaneous determination of tyrosine, guanine, adenine and thymine, Carbon, vol. 45, pp. 27832796, 2007.

[3] C. Boero, et al., Highly sensitive carbon nanotube-based sensing for lactate and glucose monitoring in cell culture, IEEE Trans. NanoBiosci., vol. 10, p.1, 2011.

[4] T. Kuila, et al., Recent advances in graphene-based biosensors, Biosens. Bioelectron., vol. 26, no. 12, pp. 46374648, 2011.

[5] Z. Zhao, et al., ZnO-based amperometric enzyme biosensors, Sensors, vol. 10, pp. 12161231, 2010.

[6] F. Patolsky, et al., Fabrication of silicon nanowire devices for ultrasensitive, label-free, real-time detection of biological and chemical species, Nat. Protoc., vol. 1, pp. 17111724, 2006.

[7] X. Zhou, et al., Silicon nanowires as chemical sensors, Chem. Phys. Lett., vol. 369, pp. 220224, 2003.

[8] A. Wanekaya, et al., Nanowirebased electrochemical biosensors, Electroanal., vol. 18, no. 6, pp. 533550, 2006.

[9] J. Janata, et al., Conducting polymers in electronic chemical sensors, Nat. Mater., vol. 2, pp. 1924, 2003.

[10] Y. Cui, et al., Nanowire nanosensors for highly sensitive and selective detection of biological and chemical species, Science, vol. 293, pp. 12891292, 2001.

[11] E. Stern, et al., Semiconducting nanowire field-effect transistor biomolecular sensors, IEEE Trans. Electron Devices, vol. 55, no. 11, pp. 31193130, 2008.
[12] S. Carrara, et al., Memristive-biosensors: A new detection method by using nanofabricated memristors, Sens. Actuators B, vol. 171172, pp. 449457, 2012.

[13] D. Strukov, et al., Themissing memristor found, Nature, vol. 453, no. 7191, pp. 8083, 2008.

[14] D. Sacchetto, et al., Multiplexed electrical detection of cancermarkerswith nanowire sensor arrays, BioNanoSci., vol. 1, pp. 13, 2011.

[15] S. Carrara, et al., Stm image formation of organic thin films: The role of water shell, Physica E, vol. 16, no. 16, pp. 65776582, 2000.

[16] F. Puppo, et al., "Memristive biosensors under varying humidity conditions." NanoBioscience, IEEE Transactions on 13.1 (2014): 19-30.

[17] A. Kusnezow, et al., Antibody microarrays: An evaluation of production parameters, Proteomics, vol. 3, no. 3, pp. 254264, 2003.

[18] D. Kim, et al., Single-protein molecular interactions on polymermodified glass substrates for nanoarray chip application using dual-color tirfm, Bull. Korean Chem. Soc., vol. 28, no. 5, pp. 783790, 2007.

[19] F. Puppo, et al., "High Sensitive Detection in Tumor Extracts with SiNW-FET in-Air Biosensors." Proceedings of the IEEE Sensors 2014. No. EPFL-CONF-201901. IEEE, 2014.

[20] F. Puppo, et al., Femto-molar sensitive field effect transistor biosensors based on silicon nanowires and antibodies, in 2013 IEEE SENSORS proceedings, Nov 2013, pp. 14.

[21] F. Puppo, et al., Memristive sensor for $\mathrm{pH}$ measure in dry conditions, Surf. Sci., vol. 624, pp. 7679, 2014.

[22] E. Stern, et al., Importance of the Debye Screening Length on Nanowire Field Effect Transistor Sensors, Nano Lett., vol. 7, no. 11, pp. 34053409 , 2007.

[23] Wong, Sau C., et al., "A 5000-gate CMOS EPLD with multiple logic and interconnect arrays." Custom Integrated Circuits Conference, 1989., Proceedings of the IEEE 1989. IEEE, 1989.

[24] Yoshimoto, Masahiko, et al., "A divided word-line structure in the static RAM and its application to a 64K full CMOS RAM." Solid-State Circuits, IEEE Journal of 18.5 (1983): 479-485.

[25] Huffman, David A. "A method for the construction of minimum redundancy codes." Proceedings of the IRE 40.9 (1952): 1098-1101.

[26] Peng, Hanchuan, et al., "Feature selection based on mutual information criteria of max-dependency, max-relevance, and min-redundancy.' Pattern Analysis and Machine Intelligence, IEEE Transactions on 27.8 (2005): 1226-1238. 